\title{
Analysis and reduction of thermal magnetic noise in liquid-He dewar for sensitive low-field nuclear magnetic resonance measurements
}

\author{
S.-m. Hwang, K. K. Yu, Y. H. Lee*, C. S. Kang, K. Kim, and S. J. Lee \\ Korea Research Institute of Standards and Science, Daejeon, Korea
}

(Received 21 June 2013; revised or reviewed 25 June 2013; accepted 26 June 2013)

\begin{abstract}
For sensitive measurements of micro-Tesla nuclear magnetic resonance ( $\mu \mathrm{T}-\mathrm{NMR}$ ) signal, a low-noise superconducting quantum interference device (SQUID) system is needed. We have fabricated a liquid He dewar for an SQUID having a large diameter for the pickup coil. The initial test of the SQUID system showed much higher low-frequency magnetic noise caused by the thermal magnetic noise of the aluminum plates used for the vapor-cooled thermal shield material. The frequency dependence of the noise spectrum showed that the noise increases with the decrease of frequency. This behavior could be explained from a two-layer model; one generating the thermal noise and the other one shielding the thermal noise by eddy-current shielding. And the eddy-current shielding effect is strongly dependent on the frequency through the skin-depth. To minimize the loop size for the fluctuating thermal noise current, we changed the thermal shield material into insulated thin $\mathrm{Cu}$ mesh. The magnetic noise of the SQUID system became flat down to $0.1 \mathrm{~Hz}$ with a white noise of $0.3 \mathrm{fT} / \sqrt{\mathrm{Hz}}$, including the other noise contributions such as SQUID electronics and magnetically shielded room, etc, which is acceptable for low-noise $\mu \mathrm{T}$-NMR experiments.
\end{abstract}

Keywords: SQUID, dewar, micro-Tesla NMR, magnetic noise, thermal shield, eddy current shielding

\section{INTRODUCTION}

Magnetometer based on superconducting quantum interference device (SQUID) is the most sensitive magnetic field sensor at low frequency range [1]. One of the recent applications of SQUID is micro-Tesla nuclear magnetic resonance ( $\mu \mathrm{T}$-NMR) or magnetic resonance imaging $(\mu \mathrm{T}$ -MRI), where a measurement field as low as about $1 \mu \mathrm{T}$ is used to detect the NMR signal. To polarize the specimen, however, a pre-polarization field is needed and its field strength needs to be as high as possible to increase the NMR signal strength $[2,3]$. Also, a large-diameter pickup coil is favorable to smaller-diameter pickup coils, in terms of SNR (signal-to-noise ratio), since the larger pickup coil can sense more magnetic flux.

The liquid He dewar should have low boil-off rate (BOR) of liquid $\mathrm{He}$ and at the same time low thermal noise for sensitive NMR measurements. The BOR of liquid He depends directly on the thermal load or thermal input, which consists of conductive heat loads from neck tube and insert neck, and radiation input through vacuum space. As a first trial, we fabricated a dewar having rigid $\mathrm{Al}$ plates for the thermal shield material. In terms of BOR, Al plates provided reasonable performance. But, the magnetic noise is much higher than expected. We calculated the magnetic noise from the Al plates using a simple conductor model, and confirmed that the main noise source is due to the

* Corresponding author: yhlee@kriss.re.kr thermal magnetic noise from the $\mathrm{Al}$ plates. To reduce the noise, we selected a special thermal conductor using $\mathrm{Cu}$ mesh made of insulated $\mathrm{Cu}$ wire, and replaced the aluminum plates by the $\mathrm{Cu}$ mesh. Final test of the SQUID system showed that low-frequency noise is nearly the same as the white noise level, and the noise level became acceptable for sensitive $\mu$ T-NMR measurements. Analysis of the thermal noise, and fabrication of the dewar are described here.

\section{STRUCTURE OF DEWAR}

\subsection{Design of the Dewar}

The schematic drawing of the dewar with an SQUID insert is shown in Fig. 1. To increase the signal picked up by the pickup coil, the pickup coil diameter has been substantially increased. Through this design, the NMR sample can be placed just under the center of the pickup coil to achieve maximum signal.

The pickup coil was made of $\mathrm{NbTi}$ wire of $0.125 \mathrm{~mm}$ diameter wound on a fiberglass reinforce plastic tube with a diameter of $140 \mathrm{~mm}$. To reduce the pickup of a rather strong prepolarization field, a second-order gradiometer structure with a baseline of $83 \mathrm{~mm}$ in 1:2:1 winding configuration was chosen for the pickup coil. The SQUID sensor is a commercial DC-SQUID (model CE2Sblue from Supracon, Germany). The parameters of the SQUID gradiometer are given in Table I. The flux transformer has a 


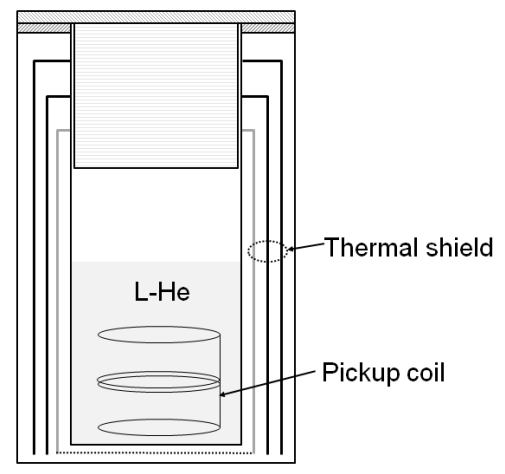

Fig. 1. Schematic drawing of the liquid He dewar and insert for the SQUID pickup coil.

TABLE I

PARAMETERS FOR SQUID GRADIOMETER.

\begin{tabular}{cc}
\hline Parameter & Value \\
\hline Input coil inductance & $420 \mathrm{nH}$ \\
Mutual inductance (input coil - SQUID) & $8.1 \mathrm{nH}$ \\
Coupling constant (input coil - SQUID) & $0.26 \mu \mathrm{A} / \Phi_{0}$ \\
Intrinsic current noise (input coil) & $<1.5 \mathrm{pA} / \sqrt{\mathrm{Hz}}$ \\
Wire diameter of pickup coil & $0.125 \mathrm{~mm}$ \\
Pickup coil diameter & $142 \mathrm{~mm}$ \\
Pickup coil inductance & $2.54 \mu \mathrm{H}$ \\
Transfer coefficient (flux transformer) & $0.048 \mathrm{nT} / \Phi_{0}$ \\
\hline
\end{tabular}

transfer coefficient of $0.048 \mathrm{nT} / \Phi_{0}$. Based on the intrinsic noise value of the SQUID given by the maker (current noise in the input coil of $1.5 \mathrm{pA} / \sqrt{\mathrm{Hz}}$ ), the estimated intrinsic field noise of the gradiometer was $0.3 \mathrm{fT} / \sqrt{\mathrm{Hz}}$ at white region, without including noise contributions of other components.

\subsection{Measurement and Analysis of Thermal Noise}

The magnetic field noise of the SQUID system was measured inside a magnetically shielded room in flux-locked loop mode, using the SQUID control electronics JESSY (from Supracon). The measured noise spectrum is shown in Fig. 2. At $1 \mathrm{~Hz}$, the field noise was 36 $\mathrm{fT}_{\mathrm{rms}} / \sqrt{\mathrm{Hz}}$, decreasing with increasing frequency.

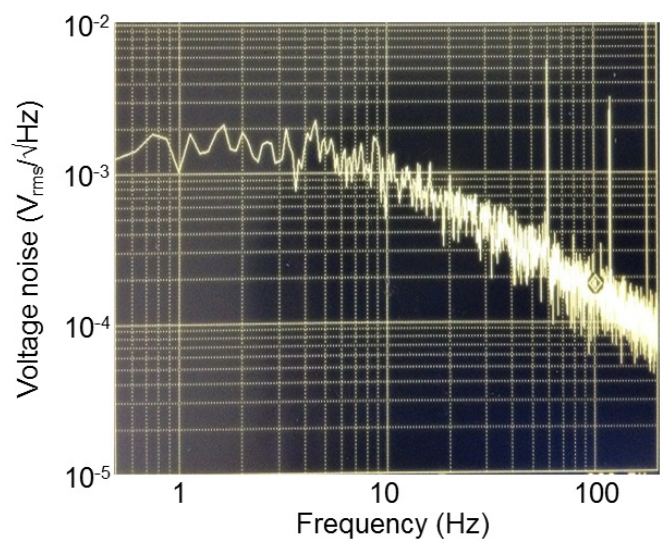

Fig. 2. Noise spectrum of the SQUID system. In the vertical axis, $1 \mathrm{~V}$ corresponds to $21 \mathrm{pT}$.

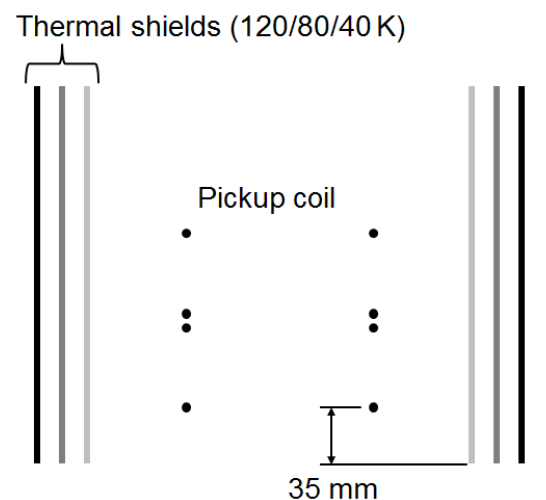

Fig. 3. Layout of the 3 thermal shields at anchoring temperatures of $120,80,40 \mathrm{~K}$, respectively. The lower end of the second-order gradiometer coil is $35 \mathrm{~mm}$ above the lower end of thermal shields.

At $100 \mathrm{~Hz}$, the noise was $3.7 \mathrm{fT}_{\mathrm{rms}} / \sqrt{\mathrm{Hz}}$. In general, the noise value was much larger than expected, and we analyzed the origin of the increase of noise at low frequency. In the vacuum space of the dewar, we installed 3 layers of aluminum tubes for vapor-cooled thermal shield, as shown in Fig. 3. The 3 shields were aluminum plates each with $1 \mathrm{~mm}$ thickness, and the shields have been anchored at the neck of the dewar at approximate temperatures of 120,80 and $40 \mathrm{~K}$, respectively.

Electrically conducting materials generate thermally induced magnetic noise due to thermal fluctuations of conduction electrons inside the conductor [4-6]. If we model a conducting cylinder as shown in Fig. 4, the field noise inside the cylinder can be expressed as the following equation [6].

$$
\begin{aligned}
B_{z}=\left(\frac{\mu_{0}}{2}\right) \sqrt{\frac{k \sigma t T}{2 \pi}} & \left\{\frac{3 \pi}{4 a^{2}}+\frac{z a}{\left(a^{2}+z^{2}\right)^{2}}\right. \\
& \left.+\frac{3 z}{2 a\left(a^{2}+z^{2}\right)}+\frac{3}{2 a^{2}} \tan ^{-1} \frac{z}{a}\right\}^{1 / 2}
\end{aligned}
$$

Here, $\mu_{0}$ is the permeability in free space $\left(4 \pi \times 10^{-7} \mathrm{H} / \mathrm{m}\right)$, $k$ is the Boltzmann constant $\left(1.38 \times 10^{-23} \mathrm{~J} / \mathrm{K}\right), \sigma$ is the electric conductivity of the metal, $t$ is the thickness of the cylinder, $T$ is the temperature, $a$ is the radius, and $z$ is the distance of the pickup coil from the lower end of the cylinder, respectively.

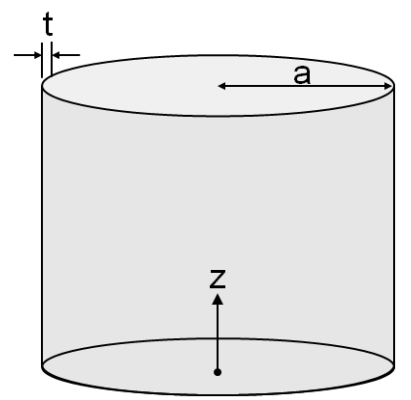

Fig. 4. Thermal conductor model of cylinder structure. 
The cylinder has radius $a$, electrical conductivity $\sigma$, thickness $t$ and temperature $T$.

To calculate noise value accurately, we need exact values of electric conductivities and temperatures of all the 3 tubes. However, the tubes have different temperature gradients along their lengths from the neck. And the temperature dependence of electric conductivity should be measured, which is beyond the scope of this work. For rough estimation of the noise, we approximated that the temperature of tube are uniform along their lengths. Though the electric conductivity of aluminum increases with decrease of temperature, we put the term $\sqrt{ }(\sigma T)$ constant for the 3 tubes of different temperatures.

Using the values of $\sigma=5 \times 10^{7}(\Omega \mathrm{m})^{-1}$ for $\mathrm{Al}, T=80 \mathrm{~K}$, $a=189 \mathrm{~mm}$, and $z=118 \mathrm{~mm}$ (center of pickup coil height from the shield tail), the calculated noise from a single tube is about $20 \mathrm{fT}_{\mathrm{rms}} / \sqrt{\mathrm{Hz}}$. Adding the other 2 tubes (by multiplying $\sqrt{ } 3$ ), the total noise becomes about 35 $\mathrm{fT}_{\mathrm{rms}} / \sqrt{\mathrm{Hz}}$, which is roughly the same value as the measured value at low frequency (Fig. 2).

\subsection{Attenuation of Noises by Inner Tube}

Next, decrease of the noise as the frequency increases should be understood. If we assume that the aluminum tubes not only generate noise but also shield noises, inner tube(s) may shield noises generated by outer tube(s). The attenuation $A$, which is the inverse of the shielding factor, can be calculated using the following equation [4].

$$
A=1 / \sqrt{1+\left(\frac{t D}{2 \delta^{2}}\right)^{2}}
$$

Where, $\mathrm{t}$ is the thickness of the cylinder, $\mathrm{D}$ is the diameter and $\delta$ is the skin depth of the conductor. At a frequency $f, \delta$ is given as following.

$$
\delta=1 / \sqrt{\mu_{0} \pi \sigma f}
$$

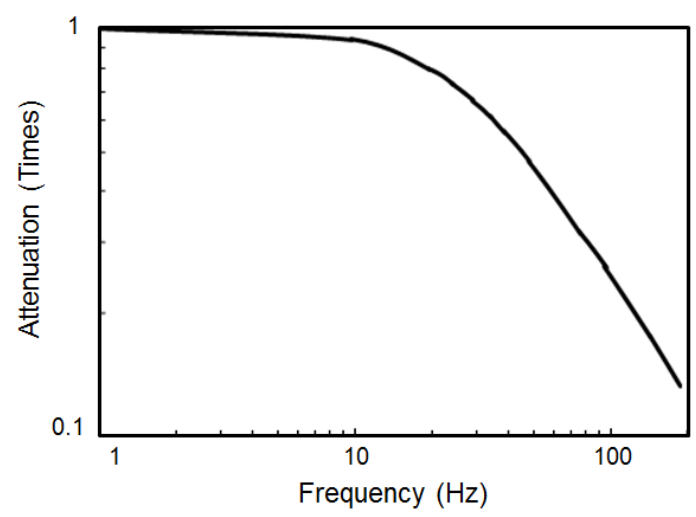

Fig. 5. Attenuation of the aluminum tube as a function of frequency.

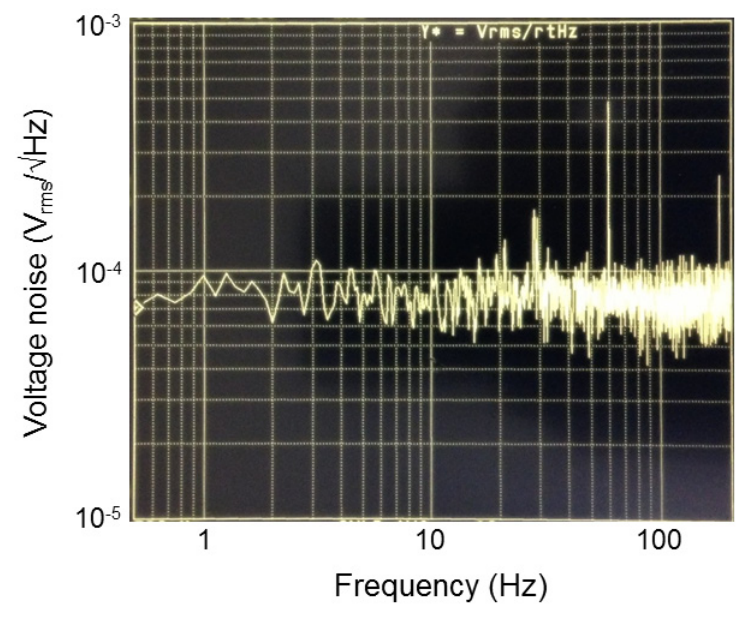

Fig. 6. Noise spectrum after modification of the vapor cooled thermal shield. $1 \mathrm{~V}$ corresponds to $21 \mathrm{pT}$.

The attenuation as a function of frequency is shown in Fig. 5, for the innermost tube of $1 \mathrm{~mm}$ thickness. Though the thickness of the aluminum tube is much less than its skin depth (for example, $7 \mathrm{~mm}$ at $100 \mathrm{~Hz}$ ), the attenuation occurs already at a frequency of around $10 \mathrm{~Hz}$. The shape of the attenuation curve is quite similar to the decrease of noise with frequency in Fig. 2, explaining that the inner aluminum tube acts as a shield of thermal noise from outer tubes in the frequency range over $10 \mathrm{~Hz}$.

To reduce the thermal noise from the aluminum tubes, we replaced the tube material with thin flexible copper mesh insulated with urethane coating (Taiyo Wire Cloth, Japan). The wire diameter was $0.08 \mathrm{~mm}$ and mesh number was 100. All 3 thermal shield layers were replaced by the copper meshes. After reassembly, the noise spectrum was measured again and the result is shown in Fig. 6. The curve is flat down to $0.5 \mathrm{~Hz}$, and the white noise was measured at $1.68 \mathrm{fT}_{\mathrm{rms}} / \sqrt{\mathrm{Hz}}$, which is quite acceptable for low-noise $\mu \mathrm{T}-\mathrm{NMR}$ measurements.

\section{CONCLUSIONS}

For mechanical stability, we used thick aluminum plates for the material of vapor cooled thermal shield. But, large thermal noises generated by the aluminum tubes made the SQUID system not acceptable for low-noise $\mu \mathrm{T}$-NMR measurements. Estimation of thermal noise value and frequency dependence was done using a simple conductor model. Replacing the aluminum plates by insulated copper mesh resulted in the white noise reduction to 1.68 $\mathrm{fT}_{\mathrm{rms}} / \sqrt{\mathrm{Hz}}$ and the noise level was flat down to $0.5 \mathrm{~Hz}$, proving that this SQUID system with a large-diameter $2^{\text {nd }}$-gradiometer pickup coil can be used for sensitive $\mu \mathrm{T}-\mathrm{NMR}$ measurements.

\section{ACKNOWLEDGMENT}

This work was supported by the Korea Research Institute 
of Standards and Science under the project "Development of Biomagnetic Resonance measurement technology (13011076)".

\section{REFERENCES}

[1] J. Clarke, and A. Braginski, "The SQUID Handbook," Wiley-VCH, 2006.

[2] H. C. Seton, J. M. S. Hutchinson, and D. M. Bussel, "A $4.2 \mathrm{~K}$ receiver coil and SQUID amplifier used to improve the SNR of low-field magnetic resonance images of the human arm," Meas. Sci. Tech., vol. 8, pp. 198-207, 1997.
[3] R. McDermott, N. Kelso, S-K. Lee, M. Mößle, M. Mück, W. Myers, B. ten Haken, H. C. Seton, A. H. Trabesinger, A. Pines and J. Clarke, "SQUID detected magnetic resonance imaging in microtesla magnetic fields," J. Low Temp. Phys., vol. 135, pp. 793, 2004.

[4] G. Stroink and C. MacAulay, "Thermal magnetic noise generated by an eddy current shielded room," Rev. Sci. Instrum., vol. 57, no. 4 pp. 658-660, 1986.

[5] S. Uzunbajakau, A. P. Rijpma, J. Dolfsma, H. J. G. Krooshoop, H. J. M. ter Brake, M. J. Peters, and H. Rogalla, "Magnetic flux fluctuations due to eddy currents and thermal noise in metallic disks," IEEE Trans. Magn., vol. 39, no. 4, pp. 2018-2023, 2003.

[6] N. Kasai, K. Sasaki, S. Kiryu, and Y. Suzuki, "Thermal magnetic noise of dewars for biomagnetic measurements," Cryogenics, vol. 33, pp. 175-179, 1993. 